\title{
Budapest, 1956: Adolfo Sánchez Vázquez, de la lingüística a la estética
}

\section{Budapest, 1956: Adolfo Sánchez Vázquez, from Linguistics to Aesthetics}

\author{
César A. Núñez \\ Universidad Autónoma Metropolitana-Iztapalapa \\ laorillayyo@yahoo.com \\ orcid.org/oooo-ooo2-6620-3682
}

Resumen: El artículo analiza un escrito olvidado de Adolfo Sánchez Vázquez publicado en la revista Nuestro Tiempo en 1953. En ese ensayo se reconoce una determinada noción de lenguaje que permitiría rehabilitar aquella literatura ajena a la poética realista. Sin embargo, los cambios - políticos, estéticos, conceptuales-operados al momento de iniciar la escritura de su famoso libro Las ideas estéticas de Marx (1965) conllevan el abandono de esa atención al lenguaje y acarrean una serie de conflictos que merecen ser considerados. La yuxtaposición entre los dos momentos de la obra de Sánchez Vázquez ilumina problemas críticos y teóricos que están en el núcleo de la disciplina estética.

Palabras clave: literatura, marxismo, política, Adolfo Sánchez Vázquez

Abstract: The paper analyzes a forgotten essay by Adolfo Sánchez Vázquez, published in the magazine Nuestro Tiempo in 1953. This article recognizes a special notion of language that would allow the rehabilitation of a literature that is far from the realistic poetics. However, the changes - political, aesthetic, and conceptualproduced when he was initiating the writing of his famous book The Aesthetic Ideas of Marx (1965) involve the abandonment of that attention to language and bring a series of conflicts that deserve to be considered. The juxtaposition of these two moments of Sánchez Vázquez's work illuminates critical and theoretical problems that are at the core of the aesthetic discipline.

Keywords: literature, marxism, politics, Adolfo Sánchez Vázquez

Recibido: 1 de agosto de 2019

Aceptado: 28 de octubre de 2019 
Nunca fueron tan silenciosas las aves Aralia López González

Iósif Vissariónovich Dzhugashvili, más conocido como José Stalin, murió en Moscú el 5 de marzo de 1953, abandonando así — por causas de fuerza mayor, es claro- el cargo de presidente del Consejo de Ministros de la Unión Soviética. En julio de ese mismo año, el número 9 de Nuestro Tiempo, que se imprimía en México, se dedica casi por completo al líder soviético. La noticia de la muerte de Stalin, en verdad, ya se tenía y había sido publicada en el número anterior, el octavo, de marzo, pero entonces habrá tomado a la redacción con la composición muy avanzada de aquella entrega, al punto de que no llegó a darle tiempo para dedicarla al dirigente fallecido. No obstante, la importancia del suceso debió mover a los responsables de la revista a incluir, de última hora, una suerte de pequeño encarte inicial: ocho páginas sin numerar a continuación del índice y antes de la página que ostenta el número 1, en las que se imprimen dos escritos sobre el fallecimiento de Stalin: una nota editorial, “¡Ha muerto el camarada Stalin!” (Anónimo 1953a: s. n. p.), y un poema de Rafael Alberti: "Redoble lento por la muerte de Stalin" (escrito en Buenos Aires el 9 de marzo, por lo que rápidamente se difundió entre los círculos del Partido Comunista de habla hispana), precedidos ambos por un solemne retrato del "hombre de acero".

La revista Nuestro Tiempo, subtitulada "Revista Española de Cultura", había sido fundada en México, en julio de 1949, por el Partido Comunista de España en el destierro. La dirigió Juan Vicens y el comité de redacción contó con destacados comunistas del exilio, como Julio Luelmo - que fue secretario de redacción-, José Ignacio Mantecón, José Renau, Miguel Prieto o Wenceslao Roces (con quien, por cierto, Sánchez Vázquez traduciría del ruso, pocos años después, tres títulos para Grijalbo), ${ }^{1}$ amén de colaboradores como Juan Rejano, Pedro Garfias y españoles refugiados en otros países: César M. Arconada, Juan Chabás, José Herrera Petere (ya radicado en Ginebra), Jorge Semprún, Rafael Alberti, etc. En Nuestro Tiempo, como dicen Yolanda Blasco Gil y To-

1 Dos libros de F. V. Konstantinov (El materialismo histórico, en 1957, y Los fundamentos de la filosofía marxista, título queen 1959 inicia la colección "Enciclopedia de la filosofía”, del que hubo una segunda edición corregida y aumentada según nueva edición rusa, en 1965, y que fue editado también en La Habana en 1964) y otro de Mark M. Rosental y G. M. Straks (Categorías del materialismo dialéctico, 1958). Años más tarde, Sánchez Vázquez publicará tres textos dedicados al famoso traductor de $E l$ Capital (1991, 1992a y 1992b). 
más Saorín Pérez, "las cuestiones culturales estaban relacionadas con temas políticos y doctrinales" (2014:311). En efecto, la revista había aparecido como consecuencia de la política cultural diseñada en el Congreso Mundial de Partidarios de la Paz (el primer número de la primera época, de hecho, estaba en gran parte dedicado al evento, "por estimarlo de fundamental interés para la causa de la liberación de España y los problemas vivos de su cultura” [Anónimo 1949: 5] $)^{3}$ y seguía los dictados estéticos del XVII Congreso del PC ruso —el famoso congreso donde se "canonizó" el realismo socialista y donde, en enero de 1934, se había fundado la Unión de Escritores-. Nuestro Tiempo, justamente, divulgaba artículos de algunos miembros de esa Unión de Escritores, personalidades oficiales como Andrei Zhdanov o Alexander Fadeev, 4

2 Sobre la revista, véase Francisco Caudet 2007: 415-428. Hay también un artículo de Teresa Férriz Roure, cuya autoría y cuyo título dejan entrever su interés ("Nuestro Tiempo (1949-1953), una publicación del Partido Comunista de España en el exilio mexicano", 1999), que por desgracia no he podido conseguir ni consultar.

3 En el marco de la guerra de Corea, la Kominform difundió la "lucha por la paz" como estrategia frentista para aglutinar posiciones prosoviéticas y "se transformó en el órgano directivo del Movimiento por la Paz, ampliando su esfera de influencia hacia grandes sectores de la opinión pública, en un doble esfuerzo por recuperar la influencia en las organizaciones de masas y salir de su aislamiento. Cuando se celebre en la martirizada ciudad polaca de Weoclaw el Congreso Mundial de la Paz, Moscú habrá dado el primer paso en lo que fue su más exitosa iniciativa frentista y su única estructura internacionalista por las siguientes décadas. Apelando a un asunto ampliamente emocional, los comunistas volvieron a convocar a los intelectuales a cumplir un papel principal en la cruzada contra una nueva guerra, el peligro atómico y la defensa de la URSS, ahora amenazada, afirmaban, por la vocación expansionista del imperialismo norteamericano. Reavivando los tópicos del antifascismo, el movimiento pacifista se valió tanto de una sensibilidad preexistente como de sus estructuras de participación. Los encuentros, las conferencias, los petitorios se multiplicaron por todos los países y muchos intelectuales occidentales estuvieron dispuestos a olvidar las purgas y los campos de trabajo para poner su nombre y prestigio a disposición de la cruzada pacifista" (Petra 2013: 104-105). Los congresos en Wroclaw (verano de 1948) y Budapest (diciembre de 1948) "fueron el primer eslabón de un amplio y vasto movimiento que aglutinó a miles de hombres y mujeres de todo el mundo tras las banderas de la defensa de la paz y la cultura contra las amenazas del 'nuevo fascismo' norteamericano. Calificado desde un principio por la prensa liberal de ser una 'fachada comunista' dispuesta para explotar las buenas intenciones de intelectuales incautos, lo cierto es que el Movimiento por la Paz despertó la adhesión de escritores, científicos y artistas de gran prestigio y fue la iniciativa cultural más exitosa impulsada por la URSS en los años de la Guerra Fría” (Petra 2013: 105).

4 De Andrei Zhdanov se imprime - en el primer número de la segunda época, en septiembre de 1951- "Sobre la literatura", "su discurso pronunciado en el Primer Congreso de Escritores Soviéticos, el 17 de agosto de 1934”, del que la redacción aclara que "aún no había sido traducido a nuestro idioma"; de A. Fadeev, aparecen 
además de imprimir trabajos de otros escritores del campo soviético, como el temible crítico Vladmir Ermilov, por ejemplo, o prestigiosos autores del bloque oriental, como György Lukács, un filósofo con el que Sánchez Vázquez presenta mayores vínculos de los que podrían pensarse a primera vista.

Sánchez Vázquez ya había publicado, en el número 5 de la revista, de enerofebrero de 1952, un artículo sobre "Antonio Machado, su poesía y su España" (1952a: 7-12) y un “Romance español de Lenin” (1952b: 68-69) —único poema suyo, por lo que sé, que no fue recopilado en su Poesía (2005c) -, escrito al calor del "XXVIII aniversario de la muerte de Lenin". Aznar Soler lo considera "tributo inevitable de la musa comunista de entonces a sus profundas convicciones políticas en aquellos años difíciles de la llamada 'Guerra fría'” (Aznar 2008: 39); en él, el poeta, so pretexto de cantar a Lenin, no se priva de deslizar alguna loa a Stalin:

\section{[...] La nave que tú dejaste, navega contra los vientos. Buen capitán la conduce}

\footnotetext{
"Humanismo contra la guerra" (en el primer número de la primera época, de julio de 1949) y, bajo el título "El trabajo del escritor", el "extracto de una conferencia pronunciada ante los estudiantes del Instituto Gorki de Literatura" (en el núm. 3 de la segunda época, de noviembre de 1951). En la antología sobre Estética y marxismo que Sánchez Vázquez preparó en 1970, incluyó dos escritos de Zhdánov - junto con Lukács, es el único autor de los publicados en Nuestro Tiempo que recupera allí-y en la nota biográfica correspondiente lo presenta como "Dirigente político soviético. Nació en 1896 y murió en 1948. En 1917 tomó parte en la Revolución de Octubre en el Ural. Desde 1930 miembro del cc del Pcus. De 1934 a 1944, secretario de la organización comunista de Leningrado, reemplazando al asesinado Kirov. En 1939, fue elegido miembro del Buró Político del PCUs. Desde 1944 hasta su muerte trabajó como secretario del cc del PCUS. Zhdánov desempeñó un papel importante en la formulación y realización de la política del partido y del Estado soviético, inspirada por Stalin, en el terreno del arte y de la literatura. En 1934 intervino en el Primer Congreso de Escritores Soviéticos en nombre del Pcus y del gobierno soviético; en su discurso fijó oficialmente las tareas de la literatura soviética y expuso los principios del realismo socialista como método de la literatura, la crítica y el arte soviéticos. En el periodo de la posguerra, durante los años 1946-1948, Zhdánov, como secretario del cc pronunció sendos informes sobre literatura, ante una asamblea de escritores de Leningrado, y sobre música, ante un grupo de compositores en Moscú. En ambas ocasiones reafirmó con mayor fuerza aún la política del partido y del Estado soviético frente a las deformaciones formalistas y cosmopolitas que, a juicio suyo, se manifestaban entre los escritores y compositores soviéticos. Las ideas de Zhdánov fueron recogidas en las Resoluciones del Comité Central del PC (b) de la URSS sobre cuestiones de literatura y arte de aquellos años" (Sánchez Vázquez 1970: 494-495).
} 


\begin{abstract}
tocando seguro puerto.
Sobre el mar, sobre la noche, contra remolinos ciegos, contra tormentas de sangre, Stalin, pulso de acero, va señalando la estrella que orienta hacia lo eterno. $[\ldots]$
\end{abstract}

(Sánchez Vázquez 1952b: 69).5

Ya en el número 9, el último de la "segunda época", la figura de Stalin se convierte en tema monográfico y asunto principal de la entrega. Junto con la nota editorial, titulada "Stalin y el pueblo español" (Anónimo 1953c: 1-4), entre los títulos dedicados a su memoria se imprimieron un poema de Juan Rejano (“En la muerte de Stalin”, 1953: 5-8); un texto de Gabriel García Narezo sobre "Stalin y su devoción por el hombre y el pueblo" (9-15); un estudio de Ángel Sánchez "Acerca de la obra de Stalin 'Problemas económicos del socialismo en la U.R.S.S.” (30-43); una selección de escritos sobre el arte y la cultura ("Stalin y la cultura”, [Anónimo 1953b: 44-51]) y, además, un artículo de Adolfo Sánchez Vázquez que trata de "Los trabajos de Stalin sobre la lingüística y los problemas del materialismo histórico" (1953: 16-29).

Este último es un texto poco conocido, como algunos otros publicados por esos mismos años, puesto que Sánchez Vázquez, que recopiló gran parte de su obra dispersa, no lo incluyó en ningún volumen (tampoco Sánchez Vázquez recogerá en ningún libro otro importante artículo de 1955, ni su tesis de

5 En el mismo número, inmediatamente antes del romance de Sánchez Vázquez, se publicó otro poema de características similares: el "Canto de paz a Lenin”, de Juan Rejano. Allí también se elogia a Stalin, presentándolo como una suerte de "continuador" natural, como "discípulo" de Lenin, y se repite la imagen del líder soviético como "capitán": "En la frutal faena y en la batalla extensa, / un capitán condujo las huestes al triunfo: / Stalin, tu discípulo, tu amado y fiel Stalin” (Rejano 1952: 66). Los poemas de Rejano y Sánchez Vázquez habían sido leídos, según indica una breve nota anónima que precede el pequeño dossier sobre Lenin, en un acto realizado el 28 de enero de 1952, con el patrocinio de la Asociación de Escritores y Periodistas Españoles en México, de la Federación Española de Trabajadores de la Enseñanza (Grupo Local de México) y de la Federación Universitaria Escolar de España en México. En él también habían leído poemas, escritos para el aniversario, Pedro Garfias y Gabriel García Narezo, y su publicación se anuncia para los números sucesivos (aunque solo apareció el de Garfias [1952] en el número siguiente), y el discurso de apertura fue pronunciado por Wenceslao Roces (1952). 
maestría en Filosofía, del mismo año). ${ }^{6}$ Es comprensible esta omisión, desde luego, pues se trata de un encomio bastante efusivo7 de un escrito de Stalin titulado "Acerca del marxismo en la lingüística", aparecido originalmente en el periódico Pravda el 20 de junio de 1950 y reproducido en seguida urbi et orbi.

Si bien a los ojos actuales puede resultar algo curiosa la imagen de un jefe de Estado como Stalin, tan recordado por su intervención en asuntos más inmediatamente políticos, el texto tuvo su importancia y, también, su incidencia en el debate cultural de aquel entonces. En el mundo hispánico fue profusamente difundido por la serie de publicaciones y editoriales del PC en Hispanoamérica, como el periódico Mundo Obrero, por ejemplo, o bien en folletos sueltos, sin pie de imprenta — para distribuirlos por todo el continente- ${ }^{8}$ Una anotación de Max Aub en sus Diarios (el 25 de julio de 1950) da cuenta de la recepción inmediata del texto en México:

Curiosa coincidencia: los artículos de Stalin acerca de la lingüística y mi carta a D[ámaso] A[lonso]. Eso de que ahora salga el famoso don José hablando de esas cosas, con Corea por medio, es evidentemente grandioso... y digno de Azaña... Hay en el artículo una frase molesta acerca de los Quijotes que, por lo visto, representan lo peor para el hombre del Kremlin. Bien vistas las cosas, es natural que así sea (2000: 172-173). 9

6 La tesis de maestría en Filosofía, presentada en la Facultad de Filosofía y Letras de la UnAM, se tituló Conciencia y realidad en la obra de arte; diez años después fue publicada sin su permiso por la Editorial Universitaria de San Salvador.

7 Sin embargo, anota Aznar Soler en su estudio introductorio a Incursiones literarias (Sánchez Vázquez 2008: 39, n. 59): "Por su parte, Gregorio Morán afirma que 'el trabajo de Sánchez Vázquez es de una considerable sobriedad de adjetivos y aunque lógicamente destaca la solicitud de los dirigentes soviéticos por los problemas de la cultura, tiene un rigor inusual en la época'" (Miseria y grandeza del Partido Comunista de España, 1939-1985. Barcelona, Planeta, 1986: 218).

8 En Mundo Obrero también se publicó, poco después, la segunda entrega del artículo de Stalin: "En torno a algunas cuestiones de la lingüística" (13 de julio de 1950).

9 La "frase molesta" a la que se refiere Aub aparece en un pasaje en el que Stalin dice: "Por lo que se refiere a la estructura del idioma pushkiniano, con su sistema gramatical y su léxico fundamental, se ha conservado en todo lo esencial como base de la lengua rusa, contemporánea. Y esto es plenamente comprensible. En efecto, ¿para qué es necesario que después de cada revolución se destruyan la estructura existente del idioma, su sistema gramatical y su léxico fundamental y se les sustituya por otros nuevos, como ocurre habitualmente con su superestructura? ¿A quién le hace falta que 'agua', 'tierra,' 'montaña', 'bosque, 'pez', 'hombre', 'andar', 'hacer,' 'producir', 'comerciar', 
Testimonio de la repercusión europea del texto es su mención, más o menos socarrona, por parte de Jacques Lacan en dos de sus escritos (en 1955 y 1957). ${ }^{10}$ A pesar de que Héctor López —en un libro dedicado al segundo escrito, "La instancia de la letra en el inconsciente o la razón desde Freud"se toma bastante en serio la mención que allí aparece y, comentando en un apartado el texto de Stalin, dice que "Resulta muy curioso que Stalin tenga que salir a imponer un punto de vista contradictorio con el materialismo histórico, aproximándose al criterio estructuralista en lingüística" (López 2009: 94), sería tan difícil aceptar que el punto de vista sea "contradictorio con el materialismo histórico" como que la proximidad entre Stalin y el estructuralismo radique en algo más que la negación del carácter superestructural del lenguaje. Por lo demás, la mención de Lacan no deja de ser bastante sarcástica: "Recuérdese que la discusión sobre la necesidad del advenimiento de un nuevo lenguaje en la sociedad comunista tuvo lugar realmente, y que Stalin, para alivio de los que confiaban en su filosofía, la resolvió en estos términos: el lenguaje no es una superestructura" (Lacan 2007: 476, n. 6). Más notoria - acaso debido a su mayor cercanía cronológica con el artículo de Stalin- es la ironía en el primero de los escritos de Lacan, en el que, con sorna, dice:

etc., no se denominen agua, tierra, montaña, etc., sino de otra manera? ¿A quién le hace falta que las modificaciones de las palabras en el idioma y la combinación de las palabras en la oración se hagan, no con arreglo a la gramática existente, sino por otra completamente distinta? ¿Qué provecho obtiene la revolución con semejante trastorno radical en el idioma? La historia, por regla general, no hace nada esencial sin que sea especialmente necesario. Cabe preguntar: ¿qué necesidad hay de semejante transformación radical en el idioma si está demostrado que la lengua existente, con su estructura, es completamente apta, en lo fundamental, para satisfacer las necesidades del nuevo régimen? Es posible y necesario destruir en unos cuantos años la vieja superestructura y sustituirla por otra nueva para dar libre curso al desarrollo de las fuerzas productivas de la sociedad; pero, ¿cómo destruir el idioma existente y construir en su lugar otro nuevo en unos cuantos años sin llevar la anarquía a la vida social, sin crear una amenaza de disgregación de la sociedad? ¿Quiénes, aparte de los Quijotes, pueden plantearse semejante tarea?” (Stalin 1950: 7).

10 El primero, "La cosa freudiana, o el sentido del retorno a Freud en psicoanálisis", había sido originalmente una conferencia pronunciada en la clínica neuropsiquiátrica de Viena el 7 de noviembre de 1955 y, ampliado, apareció en L'Évolution psychiatrique, núm. 1, 1956: 225-252. El segundo, titulado "La instancia de la letra en el inconsciente o la razón desde Freud”, fue un texto leído el 9 de mayo de 1957 en el anfiteatro Descartes de la Sorbona, por petición del grupo de filosofía de la Fédération des étudiants ès Lettres e impreso en el volumen 3 de la Psychanalyse, sobre el tema Psychanalyse et Sciences de l'homme, PUF, 1957: 47-81. Ambos se encuentran recopilados en el primer tomo de los Escritos de Lacan (2007). 
"No hay habla sino de lenguaje", esto nos recuerda que el lenguaje es un orden constituido por leyes, de las cuales podríamos aprender por lo menos lo que excluyen. Por ejemplo que el lenguaje es diferente de la expresión natural y que tampoco es un código. Que no se confunde con la información, metan las narices en la cibernética para saberlo; y que es tan poco reducible a una superestructura que hemos visto al materialismo mismo alarmarse de esa herejía, bula de Stalin citable aquí (2007: 396).

\section{Quien sí se toma muy en serio a Stalin es Sánchez Vázquez. El artículo} de Stalin partía de una constatación formulada en una entrevista, resultado de la cual se abandonaba buena parte de la lingüística soviética "oficial" que se hacía hasta entonces. Por un lado, negaba que el idioma fuera una superestructura sobre la base. Con ello, por otro, quedaba invalidada la posibilidad de atribuirle un carácter de clase. El asunto, desde ya, en la Unión Soviética de aquel entonces, no carecía de importancia política, y de allí la dedicación de Stalin al tema. En su artículo, Sánchez Vázquez resume la posición de Nikolái J. Marr, ${ }^{11}$ el modo en que Stalin revisa la noción de superestructura,

11 Aunque no cuenta, claro, pues es asunto ajeno a su tema y quizá a su conocimiento en aquellos años, las rencillas políticas en torno a las cuales se dirimió la lingüística soviética de entonces. Dice Jean-Jacques Marie: "Después de la literatura, el cine, la biología y la historia, la lingüística parecía preparada para sufrir la campaña de rectificación iniciada en 1946. La victoria de Lyssenko en 1948 da alas a los discípulos del lingüista proletario Marr, muerto en 1934. El 22 de octubre de 1948, el Consejo Científico del Instituto de la Lengua en Leningrado, en una disposición comunicada a Stalin, condena a ocho lingüistas acusados de 'reflejar servilmente las teorías podridas del saussurianismo y del estructuralismo' y de 'luchar contra la lingüística materialista soviética'; y pide 'medidas concretas destinadas a aplastar la lingüística reaccionaria idealista'. Uno de los ocho lingüistas denunciados, el georgiano Tchikobava, había escrito a Stalin denunciando el destrozo de la lingüística soviética a manos de los marristas. En marzo de 1950, Stalin lo convoca a su villa. El 10 de abril le ordena que escriba un artículo que discute detalladamente. [...] El artículo de Tchikobava, publicado en Pravda el 9 de mayo de 1950, suscita una viva controversia en las columnas del diario hasta producir una auténtica tormenta. El 20 de junio, Pravda publica un artículo de Stalin, 'A propósito del marxismo en lingüística', seguido muy pronto de 'Algunas cuestiones de lingüística', y por último, el 2 de agosto, de una 'Respuesta a los camaradas' (siguen cuatro nombres, entre ellos, el de un tal Jolopov, que había osado advertir a Stalin que en 1950 escribía lo contrario de lo que había escrito en 1930 sobre la fusión de las lenguas). Mientras tanto, Pravda publica unas autocríticas de marristas, convencidos de que el plan de aplastamiento de sus adversarios se volverá contra ellos. Así, Tchemodanov se extasía flagelándose en Pravda del 23 de mayo: 'El último genial trabajo del camarada Stalin es un giro en la evolución de las ciencias sociales [...], la exposición más notable, más completa y más sistemática del marxismo en el ámbito de la lingüística'. [...] Fingiendo abrir 
y glosa con tino la tesis defendida por Stalin: "Stalin se opone así a una vieja deformación del marxismo, que consistía en vincular, de modo directo, la superestructura al estado de las fuerzas productivas. Esa deformación se manifestaba, por ejemplo, en el intento de explicar directamente la literatura de una época por el nivel de desarrollo de las fuerzas productivas, en lugar de buscar dicha explicación en el análisis de la base, en el régimen social y conflicto de clases de la época dada" (Sánchez Vázquez 1953: 20). Resulta evidente que Sánchez Vázquez avizora, en el texto de Stalin, la posibilidad de disponer de una categoría que, mediando entre lo real y lo simbólico, permita relativizar la demanda que la estética soviética ortodoxa hacía sobre la literatura y rehabilitar formas literarias que, de otra manera, resultaban condenadas como decadentes o "burguesas".

En efecto, dice Sánchez Vázquez que: "Al señalar el carácter específico del idioma, Stalin dejó el camino abierto para que se investigara el de otros fenómenos sociales, hasta entonces incluidos esquemáticamente en la superestructura, y en cuyos rasgos distintivos no se había reparado suficientemente" (1953: 22). Se abre así la oportunidad de revisar la situación de disciplinas

una discusión libre sobre lingüística, persigue un doble objetivo: el primero es de fachada, el segundo más profundo. En primer lugar, reprocha a los lingüistas que no se hayan preocupado de elaborar una gramática, un diccionario y manuales de los que carecen las aproximadamente 6o lenguas y dialectos hablados en la URSS, y al mismo tiempo, al no hacerlo, de no preparar las condiciones para hacer del ruso una lengua 'zonal' dominante, en espera de ser la única en todo el territorio de la URSS. El debate sobre la lingüística es un elemento de la política de rusificación impulsada por Stalin desde 1945 a un ritmo incesantemente acelerado. Persigue un objetivo aún más oscuro: Stalin avanza siempre disfrazado. En su último texto del 4 de agosto, afirma como de pasada: la tesis de Engels según la cual, 'después de la victoria de la revolución socialista, el Estado ha de debilitarse', es falsa. Solo pueden creer en ella 'los escolásticos y los talmudistas'. ¿Por qué? 'Como la revolución socialista ha triunfado solamente en un país y el capitalismo domina en todos los demás, si el país de la revolución victoriosa no quiere verse aplastado por el cerco capitalista debe consolidar al máximo el Estado y su aparato, los servicios de información y el ejército'. [...] Al actuar así, Stalin critica su propia teoría del 'socialismo en un solo país': si el aparato de coerción social, que quiere reforzar al máximo, es el reflejo de la violencia exterior sobre la sociedad soviética por parte de un imperialismo ávido de reconquistar un mercado perdido; si es capaz de imponer un enorme y gravoso desarrollo del aparato del Estado, la 'sociedad socialista' autárquica sufre, pues, la imposición y la marca del mundo capitalista que lo rodea. En él, la teoría siempre es la sirvienta de la práctica. La teoría del refuerzo creciente del Estado y de sus órganos coercitivos debe justificar el reforzamiento de la represión y anunciar una nueva etapa del Gran Terror. Y hacerlo en torno a una discusión libre sobre la lingüística es uno de los ardides en los que Stalin se complace" (Marie 2003: 870-872). 
como la lógica, las ciencias sociales, las ciencias de la naturaleza (física, química, botánica, geología, biología, etc.) y, de manera fundamental, de revisar la situación del arte:

El problema de las relaciones del arte con la superestructura se esclarece partiendo también de las tesis de Stalin. Aunque, en general, se admite que el arte forma parte de la superestructura ya que tiene carácter de clase, surge la cuestión de qué determina el que las obras de Cervantes, Tolstoi o Miguel Ángel, por ejemplo, no solo hayan sobrevivido a los cambios de la base, sino que hoy, sobre todo en la Unión Soviética, sean patrimonio vivo del pueblo.

El problema de la supervivencia de la obra de arte hay que verlo a la luz de la tesis de Lenin, reafirmada por Stalin, de que los elementos valiosos de las viejas superestructuras no desaparecen, sino que se van integrando en un tesoro común, que constituye la herencia cultural de la humanidad. Lo nuevo no es, por tanto, la negación radical de todo lo pasado. [...] El arte, como la literatura del pasado, vive en la sociedad socialista, cuando cumple determinadas exigencias que aseguran su perennidad y que afectan, indisolublemente, al contenido y a la forma (1953: 24).

Esta mirada sobre la colocación del lenguaje en la tópica base-superestructura no deja de presentar problemas (y sobre ellos ha reflexionado magistralmente Augusto Ponzio en su libro sobre La revolución bajtiniana [1998]). Sin embargo, al joven Sánchez Vázquez le interesa porque abre una alternativa para la literatura. El lenguaje le ofrece acaso el mejor elemento con el que establecer una mediación entre lo real y lo simbólico y, por ello, es el mejor medio a través del cual pensar la especificidad de lo literario. Este problema reaparecerá, de manera clara, en la obra de Sánchez Vázquez, en los años posteriores, cuando abandone la ortodoxia estalinista.

Pero si es de celebrar la aparición en la obra de Sánchez Vázquez de lo que suele llamarse un “marxismo crítico" (véase Gandler 2007), esta modificación trae aparejado el abandono —el rechazo, incluso- de su obra anterior. Con ella, queda también en el olvido la reflexión sobre el lenguaje que despuntaba en 1953. Así, la estética que Sánchez Vázquez comienza a fraguar a finales de los cincuenta - y que tiene su mejor manifestación en el libro sobre Las ideas estéticas de Marx, aparecido en $1965-{ }^{12}$ carece de un punto de apoyo para pensar lo literario, que hubiera resultado esencial.

12 Donde la ruptura es explícita: en 1965, en Las ideas estéticas de Marx, anotará: "De estas limitaciones participaban también mis trabajos estéticos en el pasado. Me refiero a mi tesis de grado Conciencia y realidad en la obra de arte (1955) y mi ensayo 
Como decía, es comprensible que abandonara un texto del que, luego de 1956, se sentiría muy lejano. ${ }^{13}$ Se trata, en primer lugar, de un abandono de carácter político. El propio Sánchez Vázquez ha contado que "a finales de los años cincuenta mi pensamiento estético conocía un viraje radical al cabo de un proceso de distanciamiento cada vez mayor respecto del marxismo 'ortodoxo' soviético provocado por ciertos acontecimientos políticos y de orden teórico" (2006: 45).

Esos acontecimientos no son un misterio. En especial, dos hechos marcan ese distanciamiento de Sánchez Vázquez, no tanto —o no en principio— de

posterior 'Sobre el realismo socialista' (Nuestras Ideas, núm. 3, 1957, Bruselas.) A partir de mi estudio sobre 'Las ideas estéticas en los Manuscritos económico-filosóficos de Marx’ (1961) orienté mis investigaciones en la dirección que ahora expongo, tratando de superar las limitaciones señaladas y de poner la estética marxista en relación con el núcleo originario -la praxis- del pensamiento de Marx" (2005b: 88, n. 19).

13 En un libro autobiográfico, Sánchez Vázquez recuerda: "Y ya en el campo de la filosofía, mi primera aportación propia fue en el ámbito de la estética con mi tesis de maestría, presentada en 1956 y titulada Consciencia y realidad en la obra de arte. // La tesis planteaba, de acuerdo con su título, el problema de las relaciones entre la consciencia del artista encarnada en su obra y la realidad. Al postular en esa relación la representación verídica de esta última, se descalificaba el arte que daba una representación fantástica, invertida o inadecuada de lo real. Esto conducía necesariamente a privilegiar una forma histórica, concreta, del arte: el realismo. // Y a él se concede gran atención en la tesis, al considerarlo como el arte por excelencia. Justamente esta identificación de arte y realismo será rechazada — como veremosen mis trabajos de estética posteriores. // Al problema del realismo se asociaba el de la ideología pues a la visión de la realidad — verdadera o falsa — se la hacía depender de la ideología que la inspiraba. // Pero al abordar esta cuestión se abordaba asimismo un problema que Marx ya se había planteado: el de la supervivencia de la obra de arte al trascender el condicionamiento histórico, social, que se pone de manifiesto sobre todo en su contenido ideológico. // La respuesta que daba entonces me sigue pareciendo hoy uno de los aspectos rescatables de la tesis. Y la respuesta se encontraba al considerar la ideología - ya caduca - que se expresa en la obra no fuera de esta sino 'integrada en la obra, en unidad indisoluble con la forma, rebasando así los límites de su época'. O sea, como diré más tarde en otros trabajos, el contenido ideológico se da ya formado en la obra. // Ahora bien, no obstante este y otros aspectos rescatables de la tesis, esta discurría por el cauce de la estética marxista dominante con su concepción del arte como reflejo y su consecuente y excluyente identificación de arte y realismo. // En un ensayo posterior — de 1957- consagrado al arte que se consideraba propio de la nueva sociedad surgida de la Revolución rusa de 1917, el realismo seguía moviéndome por el mismo cauce aunque yo trataba de darle, dentro de él, un carácter abierto frente a las deformaciones e interpretaciones estrechas y dogmáticas del 'realismo socialista'. // El cambio que pocos años después se daría en mi pensamiento estético determinó mi decisión de no publicar la tesis" (Sánchez Vázquez 2006: 43-44). 
una posición estética como de una posición política. Estos hechos tienen fecha precisa, 1956, año bisagra en la historia del comunismo. El 25 de febrero de 1956, durante el XX Congreso del Partido Comunista (el primero luego de la muerte de Stalin, pues el anterior, el XIX, se había hecho en octubre de 1952), en una sesión a puertas cerradas — vale decir, sin los jefes de los partidos hermanados- Nikita S. Kruschev pronuncia su famoso, valga el oxímoron, "discurso secreto", que se hará conocido como "Informe Kruschev", en el que reconoce y repudia los crímenes cometidos por Stalin, critica el culto a la personalidad y exonera al Partido de culpa colectiva. ${ }^{14}$ No obstante, meses después, en noviembre de 1956, las tropas rusas entran a Budapest para aplastar la tenue distancia de Moscú que se abría en Hungría con la República Popular — del 23 de octubre al 10 de noviembre- de Imre Nagy (en esa República, Lukács fungió como ministro de cultura y, resultado de esa participación, será encarcelado en Rumanía y, a su regreso, en abril de 1957, inhabilitado para intervenir en política). Pero 1956 marca, además, el inicio de la difusión de un texto fundamental en la deriva de la estética marxista (así como de la economía, la filosofía, etc.): los Manuscritos económico-filosóficos de 1844. Estos escritos juveniles de Marx se habían publicado por primera vez en 1932 (y en el equipo editor había intervenido Lukács), pero su difusión comenzó en 1956:

Un acontecimiento también decisivo para mí, no ya de orden práctico-político sino teórico fue la lectura y el estudio - un verdadero descubrimiento — de la obra juvenil de Marx, los Manuscritos económico-filosóficos de 1844 - en una mala traducción francesa pues aún no aparecía la española de Wenceslao Roces-.

Allí encontré un verdadero tesoro: no solo una concepción del hombre, de la naturaleza y de la sociedad que no correspondía a la del Marx cientifista, objetivista, determinista que el marxismo oficial ofrecía, sino, a partir de las ideas estéticas que podían rastrearse en los Manuscritos, un pensamiento estético que echaba por tierra los principios de la llamada "estética marxista-leninista" (o estética soviética dominante).

El primer fruto de esta exploración de los Manuscritos fue el ensayo publicado en 1961 en la revista Dianoia, del Centro de Estudios Filosóficos de la UNAM, con el título de "Las ideas estéticas en los Manuscritos económico-filosóficos de Marx". Este trabajo se reprodujo poco después en la Cuba revolucionaria y, más tarde, ya reelaborado, se integró, con el título de "Las ideas de Marx sobre las fuentes de lo

14 En Occidente, el informe se publica en la prensa en el mes de junio. El PC francés se niega a reconocer su autenticidad, pero el PC italiano lo difunde e inicia un proceso de reformas (el "policentrismo", ajeno a Moscú). 
estético", en mi primer libro, aparecido en 1965, Las ideas estéticas de Marx. En él, partiendo de las ideas estéticas de Marx, se somete a crítica la estética soviética del "realismo socialista" y se apunta en dirección a una nueva estética marxista" (Sánchez Vázquez 2006: 45-46).15

A partir de la lectura de los Manuscritos - y de otros textos, como las antologías de escritos marxianos sobre arte y literatura preparadas por Mijail Lifchiz-, Sánchez Vázquez irá escribiendo los seis artículos que publicará desde 1961 y recopilará, junto con otros inéditos, en el libro Las ideas estéticas de Marx, de 1965 (Sánchez Vázquez 1961, 1964a, 1964b, 1964c, 1965a, 1965b).

En los años sesenta, gracias a esas lecturas, el arte será definido, antes que nada, como praxis, y así quedará religado al trabajo, categoría fundamental —y aquí hay una explícita y definitiva oposición a la estética kantiana-. Ahora bien, al reunir, por vía de la praxis, el arte con el trabajo, se plantea el problema de su diferencia y de su función, puesto que el trabajo es definido, ya en sí mismo, como "actividad creadora" (como praxis). El arte sería, como el trabajo, un modo de objetivación (de fines, ideas o sentimientos humanos), pero un "trabajo humano superior" que "tiende a satisfacer la necesidad interna del artista de objetivarse, de expresarse, de desplegar sus fuerzas esenciales en un objeto concreto-sensible" (Sánchez Vázquez 2005b: 61). Superioridad, expresión, esencia... para ser nuevas categorías, huelen a viejo.

En esta lógica, el arte es reivindicado en la medida en que "surge para satisfacer una necesidad específicamente humana; la creación y el goce artísticos caen, por tanto, dentro del reino de las necesidades del hombre" (Sánchez Vázquez 2005b: 32), aunque a la hora de definir esas necesidades se hable de

15 Y, en 1995, Sánchez Vázquez ya había escrito: “Debo recordar que fueron escritos entre marzo y agosto de 1844; que permanecieron en estado de borrador por voluntad de su autor hasta que en 1932 se publicaron, por primera vez, en su lengua original en la edición de Obras completas de Marx y Engels que se conoce por sus siglas en alemán: MEGA. Por diversas circunstancias históricas — consolidación del estalinismo, ascenso de los nazis al poder y Segunda Guerra Mundial- esta aparición de los Manuscritos pasó inadvertida, con algunas excepciones, dentro y fuera de los medios intelectuales y políticos marxistas. Por otra parte, dado el carácter ideologizado que habría de adquirir el marxismo en el llamado 'campo socialista' y en el movimiento comunista mundial, no cabía esperar que el pensamiento de Marx que vertebra a los Manuscritos pudiera encajar en un marxismo oficial, institucionalizado, que había soterrado toda veta crítica y humanista. En ruso, solo se publicaron en 1956, después del XX Congreso de PCUS, marginados en un volumen aparte de la nueva edición de Obras completas de Marx y Engels" (Sánchez Vázquez 1995: 222). 
una "necesidad humana meramente espiritual" (Sánchez Vázquez 2005b: 39) - y el adverbio no es mío-. "[E]l trabajo y el arte no se diferencian, como pensaba Kant, porque el primero sea una actividad interesada y el segundo una actividad gratuita, o porque el trabajo busque una utilidad, y el arte el puro placer o juego; la diferencia radica más bien en el tipo de utilidad que aportan uno y otro: estrecha y unilateral, la del trabajo; general y espiritual, la del arte" (Sánchez Vázquez 2005b: 38). La idea de una utilidad "general y espiritual" no deja de ser problemática y muestra en qué medida la estética, como disciplina, tiene serios problemas para deshacerse de su lastre iluminista. Puesto que, a la hora de explicar la aparición del arte, la presunta "utilidad" tiene que diluirse y vuelve a presentarse la idea de un "excedente", de un "sobrante": "Es preciso que el trabajo alcance un cierto nivel [...] para que se puedan producir objetos que rebasan ya su función utilitaria y que, sin excluir esta, cumplen una función estética, u objetos que se liberan por completo de esa función práctica para ser, ante todo, obras de arte" (Sánchez Vázquez 2005b: 40). Vale decir, el arte es igual al trabajo, con la salvedad de que es distinto del trabajo. Autonomía y finalidad siguen siendo, después de todo, las piedras de toque con las que se empantana la estética. Del mismo modo, la evaluación del arte — actividad tan cara a la estética — reintroduce a Kant por la ventana: cuando el objeto suscita el placer estético, dice Sánchez Vázquez, "se valora, entonces, al margen de su valoración consciente utilitaria; cabe decir, desinteresadamente, si se tiene en cuenta el interés práctico-material, pero interesadamente en cuanto que el objeto interesa porque el hombre ve en él afirmada, y materializada, su potencia creadora" (2005b: 43; [cursivas en el original]). Otro tanto podría decirse sobre las cuestiones, tan importantes para Sánchez Vázquez, de la definición de realismo y de la enajenación del trabajo artístico. ${ }^{16}$

Estos vaivenes no son anomalías. Están en el núcleo mismo de la situación del arte en el mundo moderno. Resulta curioso, eso sí, que Sánchez Vázquez no haya tenido en cuenta el enorme problema que las vanguardias (entendidas en sentido estricto o, al modo en que hace Lukács, en sentido amplio:

16 Este asunto se arrastra desde la década anterior. Así también puede verse en "Tradición y creación en la obra de arte" (1955: 146-155), donde el problema es claro: si "todo arte verdadero es innovación" y, a la vez, "no se puede dejar de innovar", no habría sino arte verdadero. Pero si hay, como dice, "falsa innovación”, resulta ser que no todo arte es innovación. No parece tener, la estética de Sánchez Vázquez, salida a esta aporía. ¿Puede haber arte malo? Si hay arte malo, la poética que defiende carece de sentido; si no lo hay, la estética misma debilita su labor. 
"modernismo") proponen a la disciplina estética. Tanto como Lukács, podría haber sido interesante contar con Theodor W. Adorno, un autor marxista que, curiosamente, Sánchez Vázquez no incluye en ninguna de las antologías que preparó sobre el tema. También desestima - algo apresuradamente- la noción de particularidad de Lukács. Cabría pensar que, publicado el libro de Lukács en 1963, Sánchez Vázquez lo lee sin tanto detenimiento; pero no retoma luego esta noción ni ninguna forma lukacsiana de pensar la autonomía del arte.

En la obra de Sánchez Vázquez posterior a 1956 la particularidad de lo literario es admitida, defendida, explicada desde la idea de creación, pero esta creación es una creación lingüística y esta particularidad no parece recordarse. En ese sentido, más allá de los comentarios explícitos con los que Sánchez Vázquez se distancia de la obra del gran crítico húngaro, su mirada sobre lo literario coincide muy a menudo con la del György Lukács anterior a 1956.

De hecho, no parece casual que, en esa suerte de "ejemplificación" de su modo de análisis que incluye el libro, ${ }^{17}$ Sánchez Vázquez se dedique a $E l$ proceso de Kafka como si el idioma no fuera sino transparente - como si su texto se tratara de un caso de aquello que Paul de Man llamaba "resistencia a la teoría” (1990)—. El estudio de esa novela lo lleva, en la medida en que soslaya el lenguaje, a la diégesis. Y la diégesis, a la referencia.

Sánchez Vázquez quiere rescatar la obra de Kafka. Explícitamente, se refiere a "la trampa de ceder su obra a la burguesía, como si a Kafka se le pudiera encerrar en el marco estrecho del mundo burgués" (2005b: 137). Aunque concede que "es cierto que expresa, de un modo peculiar y genial, este mundo en descomposición”, también reconoce que "su expresión es tal que sus persona-

17 Federico Álvarez, en el prólogo a la más reciente edición del libro, lo considera "una especie de muestra práctica de crítica literaria en torno al muy controvertido conflicto entre realismo y vanguardia [...] una especie de demostración concreta, en el terreno de la crítica literaria, de la validación de las ideas de Adolfo Sánchez Vázquez expuestas en el resto del libro sobre realismo y vanguardia" (Álvarez 2005: XIV-XV). El propio Sánchez Vázquez, hablando de Las ideas estéticas de Marx, dice: "Vayamos, pues, a este libro que se divide en dos partes. La primera se titula 'En torno a las ideas estéticas de Marx y los problemas de una estética marxista'. En ella se recogen varios ensayos que responden a ese título, así como otros dos, uno sobre Kafka y otro sobre el género de la tragedia revolucionaria, con los que se ejemplifica la aplicación de las ideas sustentadas sobre el arte y la ideología. La segunda parte está dedicada toda ella, de acuerdo con su título, a la situación del arte bajo el capitalismo, a partir de la idea fundamental de Marx sobre la hostilidad del capitalismo al arte" (2005: 46; las cursivas son mías). 
jes parecen decirnos: he aquí lo que los hombres han hecho de sí mismos; he aquí cómo se deshumanizan y degradan" (2005b: 137; las cursivas son mías).

Cuando lee la novela de Kafka, su rehabilitación la convierte en una novela realista. Pareciera tratarse de un intento de invertir la carga positiva del estilo reivindicado por Lukács en “¿Franz Kafka o Thomas Mann?” (un texto de 1954 que apareció en castellano en un libro traducido en 1963 [58-112]). Pero desafiar la preferencia de Lukács es, en gran medida, aceptar la disyuntiva -y, por tanto, aceptar el régimen de clasificación que la funda-. Así, el artículo de Sánchez Vázquez se propone mostrar que "Este desdoblamiento de la existencia, que el propio Kafka experimenta dolorosamente, será [...] una de las claves para entender el destino abstracto - descarnado o deshuesado- de José K." (2005b: 135). No solo Kafka se inscribe —acaso se expresa- en José K., sino que, como dirá más adelante, puede hallarse una "fidelidad de Kafka a lo real" pues, "en efecto, el escritor checo no ha hecho sino describir unas relaciones humanas reales, propias de la sociedad capitalista en general, en la forma particular que adoptan en el Estado atrasado de la monarquía austrohúngara de su tiempo" (2005b: 139). En definitiva, "Lo que Kafka dice de la burocracia judicial, Marx lo había señalado ya como un rasgo propio de la burocracia de un Estado opresor" (2005b: 140).

Sin embargo, es patente que quedan en la mirada de Sánchez Vázquez resquemores que vienen de antaño: "de la misma manera que no podemos criticar a Kafka por presentarnos el ser irreal, abstracto y burocrático del hombre, sino por mostrarlo de un modo intemporal y abstracto, es decir, sin revelar el suelo real que engendra ese ser abstracto del hombre; no podemos criticar tampoco a Kafka por revelar la existencia humana como una existencia irracional, absurda" (2005b: 141; las cursivas son mías); solo resta continuar la frase, y reconocer que, sin duda, echa en falta la revelación del modo histórico de esa existencia absurda. Así, resulta una suerte de lamento por el hecho de que Kafka no haya comprendido el carácter "absurdo — por estéril—" de la lucha individual, lo cual no deja de resonar como un rasgo burgués del escritor checo - o acaso de la obra, pues su diferencia con el autor es tenue-.

El personaje, en fin, adquiere una carnadura que, a los ojos de la modernidad literaria, resulta demasiado muscular: "José K. encarna este punto culminante de la enajenación humana. Esta ha llegado en él a tal extremo que ya ni siquiera experimenta su existencia como una existencia desgarrada o desdoblada" (2005b: 144; las cursivas son mías). Y, por ello, Sánchez Vázquez concluye que "Justamente por haber reducido su existencia concreta a una 
dimensión abstracta puede moverse allí [como funcionario de banco] con firmeza y seguridad" (2005b: 144).

¿A qué se debe este modo de leer la novela de Kafka? ¿Cómo explicarse esta conversión de Kafka en un escritor realista? Sin duda un patrón de lectura como la estética propende a pensar de cierta manera el arte literario. Pero, más aún, el abandono de toda idea del lenguaje —un abandono explicable en términos políticos, en gran medida, pues otras nociones del lenguaje que tenía a disposición lo hubieran alejado de las posiciones que deseaba tener entonces - hace difícil disponer de una categoría que sirva de mediación entre lo ideológico y lo literario.

Se puede decir e insistir en que el arte es praxis, pero si en ningún momento se refiere el tipo de labor que hace el arte con los materiales, se corre el riesgo de convertir la afirmación en un axioma no demostrado. Sería tanto como reconocer que la carpintería es praxis, pero negarse a hablar del tipo de operación que realiza con la madera. A la hora de pensar la literatura, el gran ausente, en el libro de 1965, es el lenguaje.

El artículo de Sánchez Vázquez sobre Stalin, en fin, deja planteada una cuestión que ya no será retomada. Acaso esa cuestión, camuflada bajo los problemas políticos que determinaron decisiones sin duda encomiables, no sea irrelevante para quien busque indagar problemas estéticos o, mejor aún, los problemas de la estética, pues queda por repensar en qué medida la disciplina puede servir (o no) para estudiar la literatura del siglo xx y, en especial, esa compleja literatura que se produce a partir de las vanguardias. Desde entonces, sus vínculos con lo social —o sus distancias de lo social, si se prefiere- se presentan más áridos de lo que las nociones de creación o de participación (como propone en uno de sus últimos libros [2005a]) parecen ofrecer.

Sin duda, lo que la crítica marxista sobre la literatura ha enseñado, de manera que ya no podemos negar, es que la significación literaria es histórica. Lo que el texto de Sánchez Vázquez pone en escena es que la crítica literaria también lo es (cosa que se olvida más frecuentemente, como si la crítica fuera un discurso atemporal). Aceptar la historicidad de las categorías con que pensamos lo literario me parece fundamental para entender cómo entendemos - en eso, en gran medida, consiste el estudio humanístico: no solo en producir conocimiento sino en reflexionar sobre las condiciones de producción de ese conocimiento- $\mathrm{y}$, a la vez, para pensar en qué sentido 
nuestro vínculo con lo simbólico es problemático, conflictivo. Solo así, creo yo, estamos en condiciones de medirnos como humanos.

Posdata. Quizá no sea del todo impertinente terminar, a modo de coda, con una reflexión de otro escritor marxista — ciertamente heterodoxo, pero marxista al fin-; un autor que ya he mencionado, tan cercano en sus temas a Sánchez Vázquez, pero tan lejano a su modo de enfocar problemas similares. En 1944, una pequeña anotación de Theodor W. Adorno, hecha durante su exilio, propone un problema al que quizá no se le haya prestado la suficiente atención. Habla allí de personas, pero bien podría referirse a textos:

$\mathrm{Al}$ que, como se dice, se atiene a la praxis, al que tiene intereses que perseguir y planes que realizar, las personas con las que entra en contacto automáticamente se le convierten en amigos o enemigos. Y como consecuencia, al poner la atención en el modo como se adecuan a sus propósitos, las reduce de antemano a objetos: utilizables los unos, obstaculizadores los otros. Toda opinión discrepante aparece en el sistema de referencia de los fines ya propuestos, sin el cual no puede hablarse de praxis, como molesta oposición, como sabotaje, como intriga; y toda adhesión, aunque provenga del interés más vulgar, se convierte en estímulo, en utilidad, en credencial para la coalición. De este modo se produce un empobrecimiento en las relaciones entre las personas: la capacidad para ver a estas como tales y no como una función de la propia voluntad, pero sobre todo la capacidad de una oposición fecunda, la posibilidad de superarse a sí mismo mediante la asunción de lo contrario, se atrofian. En su lugar se instala un conocimiento de los hombres basado en juicios para el que, a la postre, el mejor es el menos malo y el peor no lo es tanto. Pero esta reacción, esquema de toda administración, y de toda "política personal", por sí sola tiende ya, antes que toda formación política de la voluntad y toda fijación de rótulos excluyentes, al fascismo (2004: 136-137).

\section{Bibliografía}

Adorno, Theodor W. (2004). Minima moralia. Reflexiones desde la vida dañada (Obra completa, 4), Rolf Tiedemann (ed.), Joaquín Chamorro Mielke (trad.). Madrid, Akal.

Alberti, Rafael (1953). "Redoble lento por la muerte de Stalin", Nuestro Tiempo, año v, núm. 8, segunda época, 1 de marzo: s. n. p.

Álvarez, Federico (2005). "Prólogo a la presente edición”, en Adolfo Sánchez Vázquez, Las ideas estéticas de Marx. México, Siglo XXI: VII-XVI.

ANónimo (1949). “Explicación al lector”, Nuestro Tiempo, año I, núm. 1, julio: 5. 
Anónimo (1953a). “¡Ha muerto el camarada Stalin!”, Nuestro Tiempo, año v, núm. 8, segunda época, 1 de marzo: s. n. p.

ANónimo (1953b). "Stalin y la cultura”, Nuestro Tiempo, año v, núm. 9, segunda época, julio: 44-51.

ANónimo (1953c). "Stalin y el pueblo español”, Nuestro Tiempo, año v, núm. 9, segunda época, julio: 1-4.

Aub, Max (2000). Diarios 1939-1952, edición, estudio introductorio y notas de Manuel Aznar Soler. México, Consejo Nacional para la Cultura y las Artes.

Aznar Soler, Manuel (20o8). "Adolfo Sánchez Vázquez, poeta, ensayista y crítico literario", en Adolfo Sánchez Vázquez, Incursiones literarias, Manuel Aznar Soler (ed.), presentación de Federico Álvarez Arregui. Sevilla, Centro de Estudios Andaluces, Consejería de Cultura, Junta de Andalucía/Renacimiento (Biblioteca del Exilio, 35): 13-54.

Blasco GIL, Yolanda, y Tomás SaOrín PéRez (2014). Las universidades de Mariano Ruiz-Funes: La lucha desde el exilio por la universidad perdida, prólogo de Aurora Díez-Canedo F[lores]. Murcia, Ediciones de la Universidad de Murcia.

CAUdeT, Francisco (2007). El exilio republicano en México. Las revistas literarias (1939-1971). Alicante, Universidad de Alicante.

DE MAN, Paul (1990). "La resistencia a la teoría”, en La resistencia a la teoría, Wlad Godzich (ed.), Elena Elorriaga y Oriol Francés (trad.). Madrid, Visor (Literatura y Debate Crítico, 4): 11-37 [ed. orig. The Resistance to Theory. Minneapolis, University of Minnesota Press, 1986].

FÉRrIz Roure, Teresa (1999). "Nuestro Tiempo (1949-1953), una publicación del Partido Comunista de España en el exilio mexicano", The International Newsletter of Historical Studies on Comintern, Communism and Stalinism, vols. IV-v, núms. 9-13: 267-282.

GANDler, Stefan (2007). Marxismo crítico en México: Adolfo Sánchez Vázquez y Bolívar Echeverría, pról. Michael Löwy, trad. Stefan Gandler, con la colaboración de Marco Aurelio García Barrios y Max Rojas. México, Fondo de Cultura Económica/ Universidad Nacional Autónoma de México, Facultad de Filosofía y Letras/Universidad Autónoma de Querétaro, Facultad de Ciencias Políticas y Sociales.

GARCía NAREzo, Gabriel (1953). "Stalin y su devoción por el hombre y el pueblo", Nuestro Tiempo, año v, núm. 9, segunda época, julio: 9-15.

Garfias, Pedro (1952). “A Lenin”, Nuestro Tiempo, año IV, núm. 6, segunda época, julio: 58-59.

LACAN, Jacques (2007). Escritos 1, trad. Tomás Segovia, revisada con la colaboración del autor y de Juan David Nadia, y nuevamente revisada por Armando Suárez. Buenos Aires: Siglo XXI, 1971, 10 $0^{\underline{a}}$ ed. corr. y aum. 1984, 25 $5^{\underline{a}}$ ed.

López, Héctor (2009). La "instancia" de Lacan. Actualidad de "La instancia de la letra en el inconsciente o la razón desde Freud”. Mar del Plata, Editorial de la Universidad Nacional de Mar del Plata (Bitácora. Cuadernos del Analista, 1).

LuKÁcs, Georg (1963). Significación actual del realismo crítico, trad. María Teresa Toral, revisada por Federico Álvarez. México, Era (2 $2^{\underline{a}}$ ed. 1967; $3^{\underline{a}}$ ed. 1974; $4^{\underline{a}}$ ed. 1977). 
Marie, Jean-Jacques (2003). Stalin, trad. Mercedes Villar Ponz. Madrid, Ediciones Palabra.

Petra, Adriana (2013). "Cultura comunista y Guerra Fría: los intelectuales y el Movimiento por la Paz en la Argentina”, Cuadernos de Historia, 38, junio: 99-130.

Ponzio, Augusto (1998). La revolución bajtiniana. El pensamiento de Bajtín y la ideología contemporánea. Madrid, Cátedra/Universitat de València (Frónesis).

Rejano, Juan (1952). “Canto de paz a Lenin”, Nuestro Tiempo, año IV, núm. 5, enerofebrero: 61-67.

REJANO, Juan (1953). "En la muerte de Stalin”, Nuestro Tiempo, año v, núm. 9, segunda época, julio: 5-8.

Roces, Wenceslao (1949). "La lucha por la paz y la liberación de España”, Nuestro Tiempo, año I, núm. 1, julio: 24-29.

Roces, Wenceslao (1952). "En el XXVIII aniversario de la muerte de Lenin”, Nuestro Tiempo, año IV, núm. 5, segunda época, enero-febrero: 56-6o.

SÁnchez, Ángel (1953). "Acerca de la obra de Stalin 'Problemas económicos del socialismo en la U.R.S.S.”, Nuestro Tiempo, año v, núm. 9, segunda época, julio: 30-43.

SÁnchez VÁzQUEZ, Adolfo (1952a). “Antonio Machado, su poesía y su España”, Nuestro Tiempo, año IV, núm. 5, enero-febrero: 7-12.

SÁNChez VÁzQUEz, Adolfo (1952b). “Romance español de Lenin”, Nuestro Tiempo, año IV, núm. 5, enero-febrero: 68-69.

SÁnchez VÁzQuez, Adolfo (1953). "Los trabajos de Stalin sobre la lingüística y los problemas del materialismo histórico”, Nuestro Tiempo, año v, núm. 9, segunda época, julio: 16-29.

SÁNCHEZ VÁzQUEZ, Adolfo (1955). “Tradición y creación en la obra de arte”, Cuadernos Americanos, año 14, vol. 84, núm. 6, noviembre-diciembre: 146-155.

SÁnchez VÁzQUEZ, Adolfo (1961). "Las ideas estéticas en los Manuscritos económicofilosóficos de Marx”, Diánoia. Anuario de Filosofía, año 7, núm. 7: 236-258.

SÁnchez VázQuez, Adolfo (1964a). "Estética y marxismo", Cuadernos Americanos, año 23, vol. 136, núm. 5, septiembre-octubre: 109-123 [ed. orig. en Unión. Revista de la Unión de Escritores y Artistas de Cuba, año III, núm. 1, enero-marzo de 1964: 8-23].

SÁNCHEz VÁzQUEZ, Adolfo (1964b). “Un héroe kafkiano: José K., Universidad de México, vol. 18, núm. 7, marzo: 28-32.

SÁnchez VÁzQUez, Adolfo (1965a). "El arte y las masas”, La Cultura en México, suplemento de Siempre!, núm. 192, octubre: 6-8.

SÁnchez VÁzQuEZ, Adolfo (1965b). "Realismo y creación artística”, Universidad de México, vol. 19, núm. 12, agosto: 4-18.

SÁnchez VÁzQUez, Adolfo (comp.) (1970). Estética y marxismo. México, Era (El hombre y su tiempo), 2 vols.

SÁnchez VÁzQuez, Adolfo (1991). "En homenaje a Wenceslao Roces”, en Elsa Cecilia Frost et al., Cincuenta años de exilio español en México. México, Universidad Autónoma de Tlaxcala/Embajada de España en México: 207-214 [ed. orig. en Revista Mexicana de Cultura, suplemento de El Nacional, vol. 2, núm. 350, 10ª época, 5 de noviembre de 1989]. 
SÁnchez VázQUez, Adolfo (1992a). "Wenceslao Roces, maestro en toda extensión de la palabra”, Gaceta UNAM, núm. 2669, 13 de julio: 1, 3.

SÁnCHEZ VÁzQUEZ, Adolfo (1992b). "Wenceslao Roces, profesor que supo dar transcendencia al conocimiento”, Gaceta UNAM, núm. 2642, 2 de abril: 12.

SÁnchez VÁzQuez, Adolfo (1995). "Los Manuscritos de 1844 de Marx en mi vida y en mi obra”, en Gabriel Vargas Lozano (ed.), En torno a la obra de Adolfo Sánchez Vázquez. México, Universidad Nacional Autónoma de México, Facultad de Filosofía y Letras: 221-236.

SÁnchez VÁzQuez, Adolfo (2005a). De la Estética de la Recepción a una Estética de la Participación. México, Universidad Nacional Autónoma de México (Relecciones).

SÁnchez VÁzQuez, Adolfo (2005b). Las ideas estéticas de Marx, prólogo de Federico Álvarez. México, Siglo XXI [ed. orig. México, Era, 1965].

SÁnchez VÁzQuez, Adolfo (2005c). Poesía, prólogo de María Dolores Gutiérrez Navas, epílogo de Adolfo Castañón. México/Málaga, Fondo de Cultura Económica/ Centro Cultural de la Generación del 27 (Letras Mexicanas).

SÁNCHEz VÁzQUEZ, Adolfo (2006). Una trayectoria intelectual comprometida. México, Universidad Nacional Autónoma de México (Relecciones).

Stalin, José (1950). Acerca del marxismo en la lingüística, s. trad. (s.p.i.).

ZhDANOv, Andrei (1951). "Sobre la literatura”, Nuestro Tiempo, año III, núm. 1, segunda época, 1 de septiembre: 55-61.

\section{César A. Núñez}

Doctor en Literatura Hispánica por El Colegio de México, profesor titular en la Universidad Autónoma Metropolitana-Iztapalapa. Es autor de Una patria allá lejos, en el pasado. Memoria e imaginación en las "Historias e invenciones de Félix Muriel" (México, 2011) y Bazar dos mundos (Buenos Aires, 2016), coautor de Una historia que no cesa. De los Centros Provinciales al Centro Galicia de Buenos Aires (Santiago de Compostela, 2014) y coordinador de Figuraciones de la escritura en la literatura hispanoamericana (Madrid, 2016). Codirige la colección Exiles and transterrados en la editorial Peter Lang y dirige la revista Signos Literarios (UAM-Iztapalapa). 